Claremont Colleges

Scholarship@ Claremont

All HMC Faculty Publications and Research

HMC Faculty Scholarship

$1-1-1984$

\title{
Uniqueness of Positive Solutions for a Class of Elliptic Boundary Value Problems
}

Alfonso Castro

Harvey Mudd College

Ratnasingham Shivaji

Southwest Texas State University

\section{Recommended Citation}

A. Castro and R. Shivaji. "Uniqueness of positive solutions for a class of elliptic boundary value problems," Proc. of the Royal Soc. of Edinburgh, 98A (1984), pp. 267-269.

This Article is brought to you for free and open access by the HMC Faculty Scholarship at Scholarship @ Claremont. It has been accepted for inclusion in All HMC Faculty Publications and Research by an authorized administrator of Scholarship @ Claremont. For more information, please contact scholarship@cuc.claremont.edu. 


\title{
Uniqueness of positive solutions for a class of elliptic boundary value problems
}

\author{
Alfonso Castro and Ratnasingham Shivaji \\ Department of Mathematics, Southwest Texas State University, \\ San Marcos, Texas 78666, U.S.A. \\ (MS received 22 December 1983)
}

\section{Synopsis}

Uniqueness of non-negative solutions conjectured in an earlier paper by Shivaji is proved. Our methods are independent of those of that paper, where the problem was considered only in a ball. Further, our results apply to a wider class of nonlinearities.

\section{Introduction}

Here we study the uniqueness of non-negative solutions to the boundary value problem

$$
\begin{array}{clll}
-\Delta u=\lambda f(u) & \text { for } & x \in \Omega, \\
u=0 & \text { for } & x \in \partial \Omega,
\end{array}
$$

where $\lambda>0$ and $\Omega$ is a bounded region in $R^{n}$ with smooth boundary.

We assume that $f(0)>0$ and that for some positive constant $C$ and some $d>1 \cdot 5$,

$$
0 \leqq f^{\prime}(u) \leqq C(1+u)^{-d}
$$

for all $u \geqq 0$. Our main result is:

THEOREM 1.1. There exists $\lambda_{0}$ such that for $\lambda \geqq \lambda_{0}$, the problem $(1.1)_{\lambda}$ has a unique non-negative solution.

Our proof depends on the maximum principle for subharmonic functions and trace inequalities (see [1]). Theorem 1.1 proves a conjecture raised in [4] where the result was proved when $\Omega$ is a ball and $d=2$.

\section{Proof of Theorem 1.1}

As is well documented (see [4]), for each $\lambda>0$, the problem $(1.1)_{\lambda}$ has a minimal and a maximal positive solution. Let them be $u_{\lambda}$ and $v_{\lambda}$ respectively. Let $w_{\lambda}=v_{\lambda}-u_{\lambda} \geqq 0$. Hence, by the mean value theorem, there exist continuous functions $\theta_{\lambda}: \Omega \rightarrow R$ such that $u_{\lambda} \leqq \theta_{\lambda} \leqq v_{\lambda}$ and

$$
\begin{aligned}
& -\Delta w_{\lambda}=\lambda f^{\prime}\left(\theta_{\lambda}\right) w_{\lambda} \text { for } x \in \Omega \text {, } \\
& w_{\lambda}=0 \quad \text { for } \quad x \in \partial \Omega \text {. }
\end{aligned}
$$

Let $z: \Omega \rightarrow R$ denote the solution of $-\Delta z=1$ in $\Omega, z=0$ on $\partial \Omega$. By the 
maximum principle, it follows that $\lambda f(0) z$ is a subsolution to $(1.1)_{\lambda}$ and $\lambda M z$ is a supersolution to $(1.1)_{\lambda}$, where $M=\sup \{f(t) ; t \in R\}$. Since, by Hopf's maximum principle, $z$ is positive in $\Omega$ and the inward normal derivative of $z$ at $\partial \Omega$ is positive, there exists constants $c_{1}>0$ and $c_{2}>0$ such inat

$$
\lambda s c_{1} \leqq \lambda z(x) f(0) \leqq u_{\lambda}(x) \leqq v_{\lambda}(x) \leqq \lambda M z(x) \leqq \lambda c_{2} s,
$$

where $s$ is the distance from $x$ to $\partial \Omega$.

Suppose Theorem 1.1 is not true. Hence, there exists a sequence $\lambda_{n} \rightarrow \infty$ such that $w_{\lambda_{n}} \neq 0$. Let $z_{n}=w_{\lambda_{n}} /\left|w_{\lambda_{n}}\right|_{2,2}$, where ||$_{2,2}$ denotes the norm in $\mathrm{H}^{2,2}(\Omega)$ (see [1]). Let $\alpha>0$ be such that if $x, y \in \partial \Omega, \xi$ and $\eta$ are the unit outward normals at $x$ and $y$ respectively, then $\{x+s \xi ; 0 \leqq s \leqq \alpha\} \cap\{y+s \eta ; 0 \leqq s \leqq \alpha\}=\varnothing$ (see [5,p. 69]). For $0<\beta \leqq \alpha$, we set

$$
\Omega_{\beta}=\Omega-\{x+s \xi ; x \in \partial \Omega, 0 \leqq s \leqq \beta, \xi \text { as above }\} .
$$

Since the boundary of the $\Omega_{\beta}$ 's is parallel to the boundary of $\Omega$, by the Trace Theorem (see [1]), there exists a constant $c_{3}$ such that

$$
\left|z_{n}\right|_{\beta} \leqq c_{3},
$$

where ||$_{\beta}$ denotes the norm in $H^{3 / 2,2}\left(\partial \Omega_{\beta}\right)$.

Now we establish an estimate for the $L^{2}$-norm of the $z_{n}$ 's on $D(\beta)=\Omega-\Omega_{\beta}$ for $0<\beta \leqq \alpha$. Let $c_{4}$ be a uniform bound for the Jacobian of the transformation $\left(x_{1}, \ldots, x_{n}\right) \rightarrow(y, s)$ with $y \in \partial \Omega$ and $s$ the component in the unit inward normal direction at $y$. Hence

$$
\int_{D(\beta)} z_{n}^{2} d x_{1} \ldots d x_{n} \leqq c_{4} \int_{0}^{\beta} \int z_{n}^{2}(y, s) d y d s,
$$

where $y$ ranges in $\partial \Omega$. By the fundamental theorem of calculus and (2.5), we have

$$
\begin{aligned}
\int_{D(\beta)} z_{n}^{2} d x & \leqq c_{4} \int_{0}^{\beta} \int_{\partial \Omega}\left(\int_{0}^{s}\left(\partial z_{n} / \partial \xi(y, t)\right) d t\right)^{2} d y d s \\
& \leqq c_{4} \int_{0}^{\beta} s \int_{0}^{s} \int_{\partial \Omega}\left(\partial z_{n} / \partial \xi\right)^{2} d y d t d s \\
& \leqq c_{3}^{2} c_{4} \beta^{3} / 3 .
\end{aligned}
$$

Let $\varepsilon<\delta<0$ and $\lambda_{n} \equiv \lambda$ be large enough so that $\lambda^{\delta}<\alpha$. By (1.2) and (2.2), we see that there exists a positive constant $c_{5}$ such that $\left|f^{\prime}\left(\theta_{\lambda}(y+s \xi)\right)\right| \leqq c_{5}(\lambda s)^{-d}$ for all $y \in \partial \Omega, 0 \leqq s \leqq \alpha$. This and (2.6) give

$$
\begin{aligned}
\int_{Z}\left|f^{\prime}\left(\theta_{\lambda}\right) z_{n}\right|^{2} d x & \leqq c_{5} \lambda^{-2 d(1+\varepsilon)} \int_{D\left(\lambda^{5}\right)} z_{n}^{2} d x \\
& \leqq c_{3}^{2} c_{4} c_{5} \lambda^{-2 d(1+\varepsilon)+3 \delta}
\end{aligned}
$$

where $Z \equiv D\left(\lambda^{\delta}\right)-D\left(\lambda^{\varepsilon}\right)$. Also from (1.2) and (2.2), we have

$$
\begin{aligned}
\int_{\Omega_{\lambda} \delta}\left|f^{\prime}\left(\theta_{\lambda}\right) z_{n}\right|^{2} d x & \leqq c_{5} \lambda^{-2 d(1+\delta)} \int_{\Omega} z_{n}^{2} d x \\
& \leqq c_{5} \lambda^{-2 d(1+\delta)},
\end{aligned}
$$


where we have used the fact that $\left|z_{n}\right|_{2,2}=1$. Hence, on combining (2.6), (2.7) and (2.8), we see that there is a constant $M>0$ such that

$$
\int_{\Omega}\left|f^{\prime}\left(\theta_{\lambda}\right) z_{n}\right|^{2} d x \leqq M\left(\lambda^{3 \varepsilon}+\lambda^{-2 d(1+\varepsilon)+3 \delta^{*}}+\lambda^{-2 d(1+\delta)}\right) .
$$

By choosing $\varepsilon=-\left(4 d^{2}+6 d\right) /\left(4 d^{2}+6 d+9\right), \delta=-4 d^{2} /\left(4 d^{2}+6 d+9\right)$, we have

$$
\left|f^{\prime}\left(\theta_{\lambda}\right) z_{n}\right|_{L^{2}(\Omega)} \leqq(3 M)^{\frac{1}{2}} \lambda^{3 \varepsilon / 2} .
$$

Thus, by a priori estimates for elliptic equations (see [2]), we have $\left|z_{n}\right|_{2,2} \leqq$ $M_{1} \lambda^{1+(3 \varepsilon / 2)}$. By our choice of $\varepsilon$ and since $d>\frac{3}{4}$ (see 1.2), we have $1+3 \varepsilon / 2<0$ which contradicts the fact that $\left|z_{n}\right|_{2,2}=1$ for all $n$, and the theorem is proved.

It is easy to see that Theorem 1.1 can be extended to more general situations such as: (i) $-\Delta$ can be replaced by any elliptic operator satisfying the maximum principle, and (ii) $f(u)$ can be replaced by $f(x, u)$ with an adequate change in (1.2).

\section{Applications}

The problem

$$
\begin{aligned}
& -\Delta u=\lambda \exp \{\alpha u /[\alpha+u] \text { for } x \in \Omega \text {, } \\
& u=0 \quad \text { for } x \in \partial \Omega \text {, }
\end{aligned}
$$

with $\alpha>0$, arises in the theory of combustion. It was proved in [3] that there is a range of $\lambda$ for which (3.1) ${ }_{\lambda}$ has at least three non-negative solutions for large values of $\alpha$. Our results prove that this range is bounded.

\section{References}

1 R. A. Adams. Sobolev Spaces (New York: Academic Press, 1975).

2 S. Agmon, A. Douglis and L. Nirenberg. Estimates near the boundary of solutions of elliptic partial differential equations satisfying general boundary conditions, I. Comm. Pure Appl. Math. 12 (1959), 623-727.

3 K. J. Brown, M. M. Ibrahim and R. Shivaji. S-shaped bifurcation curves. Nonlinear Anal. 5 (1981), 475-486.

4 R. Shivaji. Uniqueness results for a class of positone problems. Nonlinear Anal. 7 (1983), 223-230.

5 V. Guillemin and A. Pollack. Differential Topology (New Jersey: Prentice Hall, 1974).

(Issued 25 October 1984) 